E. A bel ${ }^{204}$ ), Hypochlorite und elektrische Bleiche, theoretischer T'eil; K. Drucker 205), die Anomalie der starken Elektrolyte; B. W e in s te in. Thermodynamik und Kinetik der Körper, u. a. m.

Wi e n, im April 1900.

\section{Über die Wasserstoffentwicklung im Marshschen Apparate.}

Mitteilung aus dem Institut von E. Beckman, Laboratorium $\mathrm{f}$ - angew. Chemie der Uuiversitat Lejpzig.

\section{Von Geora Lockemann.}

Bei einer Kontroverse über das geeignetste Aktivierungsmittel für Zink im $M$ a r s h schen Apparate erklärt neuerdings A. G a u t i e $\mathbf{r}^{1}$ ) wieder wie bereits vor dreißig Jahren ${ }^{2}$, daß dem Platin unbedingt der Vorzug zu geben sei, während $Z \circ 1$ t a $n$ d e $\mathrm{V}$ a m o s s ${ }^{3}$ ) bei seinen Versuchen Kupfer und Platin als gleichwertig fand, jedoch das Platin seiner schnelleren Wirkung wegen für geeigneter hält.

Demgegenüber möchte ich darauf aufmerksam machen, da $B$ ich bei meinen Untersuchungen über den Arsennachweis4) den Einfluß der verschiedenen Aktivierungsmittel auf die Arsenwasserstoffentwicklung eingehend geprüft habe und zu dem Resultat gelangt bin, daB die Arsenprobe bei Anwendung von Kupfersulfat als Aktivierungsmittel am empfindlichsten ist. Allerdings muß man das Zink vor dem Einbringen in den Apparat verkupfern und alle anhaftende Kupferlösung sorgfältig abwaschen, da sonst, wenn das Kupfersalz mit der arsenhaltigen Flüssigkeit zusammengerät, den $B e$ obachtungen $G$ a $u$ t i e rs gemäß tatsächlich ein erheblicher Teil des Arsens zurückgehalten wird. Eine analoge Erscheinung zeigt sich ja auch bei der Verwendung von Platinchlorid.

Die Verkupferung wird in der Weise ausgeführt, daß die zerkleinerten Zinkstücke (je 1,2-1,8 g) in einer Porzellanschale mit verd. Kupferlösung (1 T. Kupfervitriol, durch mehrmalige Kristallisation gereinigt, auf $200 \mathrm{~T}$. Wasser) übergossen, etwa eine Minute lang darin hin und her gerüttelt und dann mehrmals mit Wasser abgespült werden. Auf Fließpapier getrocknet, lassen sich diese schwarz überzogenen Zinkstücke in verschlossenem Gefüß für den jeweiligen Gebrauch aufbewahren.

Bei Verwendung von derart verkupfertem Zink erhielt ich noch bei $0,0001 \mathrm{mg}=0,1 \mathrm{mmg}$ As deutliche Spiegel, während bei platiniertem Zink die Grenze der Empfindlichkeit auf 0,001 $\mathrm{mg}$ $=1 \mathrm{mg}$ sank. Die Furcht $\mathrm{G}$ a $\mathrm{u}$ t i e $\mathbf{r} \mathrm{s}$, daß durch das Kupfer Spuren von Arsen mit in den Apparat geraten könnten, dürfte kaum begründet sein, da

204) Enge 1 h a r d t sche Monographien über angewandte Elektrochemie, $\mathrm{Bd}$. 17.

205) A hrenssche Sammlung chem. und chem.-techn. Vorträge, Bd. 10.

1) Bull. Soc. Chim. Paris 35, 207 (1906); Ref. diese Z. 19, 1364 (1906).

2) Annal. Chim. 9, 384 (1876).

3) Bull. Soc. Chim. Paris 35, 24 (1906).

4) Zeitschr. f. angew. Chemie 18, 416 (1905). man sowieso nur das reinste durch mehrmalige Kristallisation von etwaigen Verunreinigungen befreite Kupfersulfat verwenden kann, und da bei den hier in Betracht kommenden Mengenverhältnissen auch etwaige Spuren von Arsen weit unter der Grenze der Nachweisbarkeit bleiben. Ich habe bei ungezählten Versuchen mit verkupfertem Zink nie, ohne vorherigen absichtlichen Arsenzusatz, auch nur den Anflug eines Spiegels erhalten.

C. $\mathrm{M}$ a i und $H . H$ u $\mathbf{t}^{5}$ ) fanden unabhängig von mir ebenfalls, daß sich Kupfersulfat für die Aktivierung des Zinks besser eigne als Platinchlorid, und $H$. B. B is $h \circ p^{6}$ ), welcher meine Methode nachprüfte, gelangte bei verkupfertem Zink zu der. selben Empfindlichkeitsgrenze von $0,1 \mathrm{mmg}$.

\section{Gewinnung des Borsulfides aus Ferrobor.}

\section{Von J. Hoffmann. \\ (Eingeg. d. 6./6. 1906.)}

B e r z e l i u s stellte das Borsulfid aus elementarem Bor durch Erhitzen im Schwefeldampfe her'), W ö h l e r und D e vil l e 2 ) durch gelindes Glühen von amorphem Bor im Schwefelwasserstoffgase, F r e $\mathrm{m} \mathrm{y}^{3}$ ) aus einem Gemenge von glasiger Borsäure und Koble durch Glühen mit Schwefelkohlenstoff bei Luftabschluß.

Die einfachste Methode ist die $W$ öhler $\mathrm{D}$ e vil 1 e sche, doch kommt bei dieser Darstellung das Borsulfid sehr tever zu stehen, da das amorphe Bor kostspielig ist ${ }^{4}$ ). Das Borsulfid ist nach den von dem Verf. angestellten Versuchen aus dem viel billiger zu stehen kommenden Ferrobor ${ }^{5}$ ) herstellbar, und ist diese Methode genau so leicht ausführbar wie die Wöhler-Devill e sche.

Zur Herstellung des Borsulfides aus Ferrobor leitet man über die gepulverte Boridmasse vollkommen getrockneten Schwefelwasserstoff. Am

5) Zeitschr. f. analyt. Chem. 43, 557 (1904).

6) Journ. Americ. Chem. Soc. 28, 178 (1906). (1906).

1) B erzelius, Poggendorffs Ann. 2, 145

2) Wöh ler und Deville, Ann. de Chim. [3] 52, 90; Liebigs Ann. 105, 72.

3) Frem y, Ann. de Chim. [3] 38, 312; Compt. r. d. Acad. d. science 35, 27, 36, 178; Liebigs Ann. 84, 227; J. 1852, 341; 1853, 327 .

4) Bor hat nach H. B l ü c h e rs Auskunftsbuch für die chem. Industrie III. 1904 folgende Preise:

Bor amorph . . . . . H M 55.-; $1 \mathrm{~kg} 500 .-$ Borpulver nach Moissan H , 70.-; 1 , 600.Bor krist. . . . . . . . $1 \mathrm{~g}, 6$ 6. ; $1 \mathrm{dg} 55 .-$ Bor graphitartig. . . . $1 \mathrm{~g}, 9 .-; 1 \mathrm{dg} 80 .-$

Bor amorph nach dem Preiskurant Kahlbaum Berlin $1 \mathrm{~g}$ M 8.-

Bor amorph nach dem Preiskurant E. Merck Darmstadt 1 g M 9.-.

5) $1 \mathrm{~kg}$ Ferrobor, $20 \%$ Bor enthaltend, aus den Goldschmidtschen Thermitwerken, kommt auf M 18. - zu stehen, ist daher im Vergleiche zum amorphen Bor etwa um das Zehnfache billiger. 